\title{
Use of total cholesterol/albumin ratio as an alternative to high density lipoprotein cholesterol
} measurement

\author{
AMIN A NANJI, SUSEELA REDDY* \\ From the Division of Clinical Chemistry, Vancouver General Hospital, and the Department of Pathology, \\ University of British Columbia, and the *Metropolitan Laboratories, Vancouver, BC, Canada
}

SUMMARY In a study of apparently healthy males, we noted a correlation between serum albuminis and high density lipoprotein cholesterol (HDL-C) $(\mathrm{r}=0.32, \mathrm{p}<0.001)$. We then correlated the total cholesterol:albumin ratio (TC:Alb) with the TC:HDL-C ratio $(r=0.89, p<0.001)$. Weo used the TC:Alb ratio to determine whether this was better than TC by itself in predicting whether an individual had a TC:HDL-C ratio of $<$ or $\geqslant 5$. The ratio performed better than TCe and correctly classified $89 \%$ of individuals $(66 \%$ with TC) $(p<0 \cdot 001)$. Since measurements of TC and Alb are routinely available on multichannel analysers, use of this ratio would provide less expensive alternative to HDL-C measurement.

A wide variety of epidemiological data indicates that individual lipoproteins or combinations of lipids and lipoproteins are better predictors of risk for coronary heart disease (CHD) than are the levels of total plasma lipids alone. ${ }^{1-3}$ However, the main concern to physicians are patients whose blood lipids do not place them in the category of hyperlipidaemia but rather in a group who have "high normal" blood lipids. Of the CHD in the general population, 75\% come from this group of "high normals". ${ }^{4}$ Because persons with appreciably raised concentrations of high density lipoprotein (HDL) have mild hypercholesterolaemia, ${ }^{5}$ it is important to identify these subjects since they are in fact "protected" against atherosclerosis; and their hypercholesterolaemia should not be treated. Identification of these individuals requires determination of HDL cholesterol (HDL-C). Because HDL-C measurements are expensive, a cheaper alternative would considerably reduce medical costs. In a study of apparently healthy males, we noted a correlation between serum albumin and HDL-C concentrations $(r=0.32, p<0.001)$. Because of this observation, we attempted to determine whether the total cholesterol: albumin (TC:Alb) ratio (a) correlated with the TC:HDL-C ratio and (b) could be useful clinically. An excellent correlation was obtained between TC:Alb and TC:HDL-C $(r=0.89$, $\mathrm{p}<0.001)$. Using the TC:HDL-C as an index of cardiovascular risk, the TC:Alb ratio separated the patients with normal $(<5)$ and increased $(\geqslant 5 \Phi$ TC:HDL-C ratio better than TC by itself.

Since measurement of TC and albumin are routinely available on multichannel analysers, use of the TC:Alb ratio could considerably reduce the number of HDL-C measurements necessary.

\section{Patients and methods}

Blood samples were obtained from 122 men (agê range 42-59 yr) referred to the Metropolitar laboratory for a "health check-up." All were ifo apparently good health. Blood for analysis wass drawn by venepuncture from the subjects after they. had fasted for 12-14 h overnight. Patients with 8 chylomicron band seen on visual inspection of serum were excluded. Serum cholesterol and albumin were measured by an automated multiple analysis systema (Technicon SMA-12 Analyser). HDL-cholestere was measured after precipitation of lipoproteing containing apo B with heparin and manganesథ chloride. ${ }^{\circ}$ The range of values in the subjects for each parameter studied were: total cholestero 101-329 mg/dl, HDL-cholesterol $29-86 \mathrm{mg} / \mathrm{dl}$ an albumin $3.4-5.6 \mathrm{~g} / \mathrm{dl}$. Separation of subjects wit normal $(<5)$ and increased $(\geqslant 5)$ TC:HDL-C ratiog was done using (a) TC and (b) TC:Alb ratio. The overall percentage of patients correctly and incore 


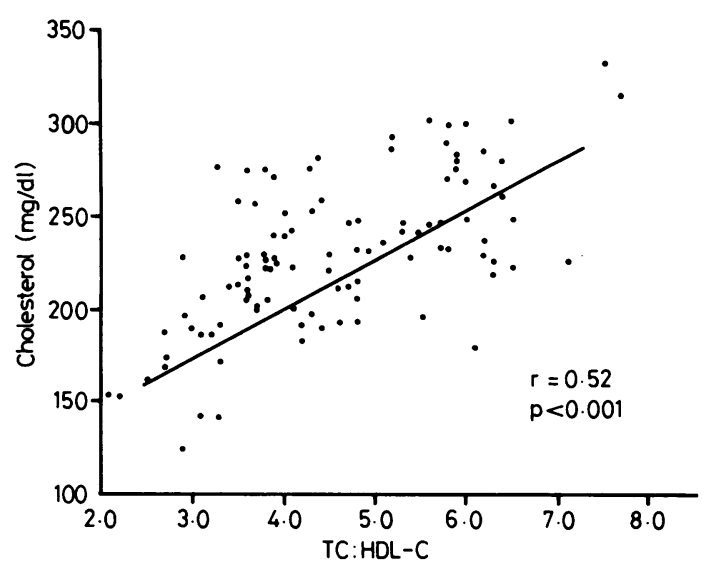

Fig. 1 Correlation between total cholesterol and TC:HDL-C ratio.

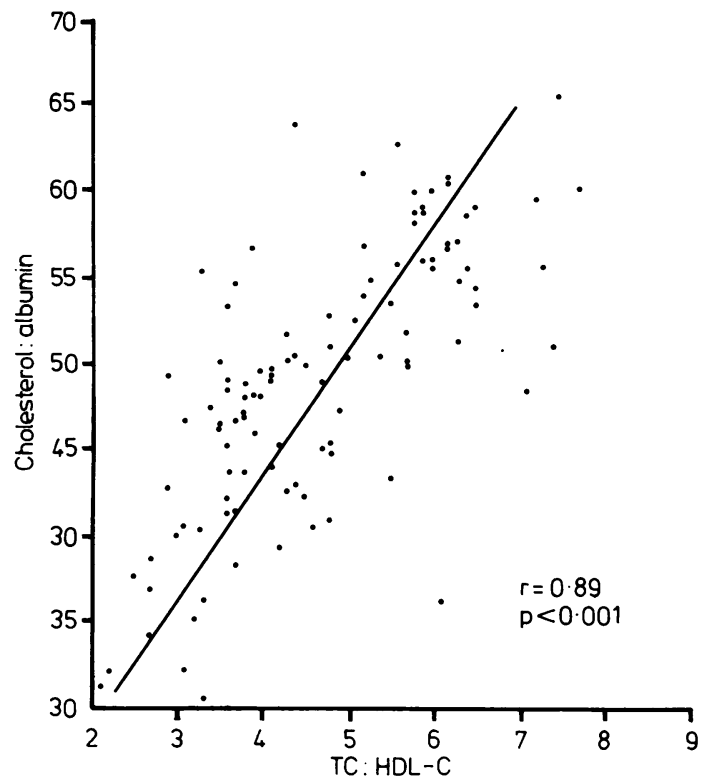

Fig. 2 Correlation between cholesterol:albumin ratio and TC:HDL-C.

rectly classified was calculated. The statistical significance of the differences between percentages was calculated using the $\chi^{2}$ test. Pearson's correlation coefficient $r$ was used to identify associations.

\section{Results}

Figure 1 shows the correlation between plasma TC and TC:HDL-C ratio $(r=0.52, p<0.001)$. Figure 2 shows the correlation between TC:Alb and TC:HDL-C ( $\mathrm{r}=0.89, \mathrm{p}<0.001)$.
Table 1 Comparison of total cholesterol (TC) and total cholesterol:albumin (TC:Alb) ratio in patients with normal and increased TC:HDL-C ratio

\begin{tabular}{|c|c|c|c|c|}
\hline \multirow[t]{2}{*}{$T C: H D L-C$ ratio } & \multicolumn{2}{|c|}{$\begin{array}{l}\text { No of subjects } \\
\text { with TC }(\mathrm{mg} / \mathrm{dl})\end{array}$} & \multicolumn{2}{|c|}{$\begin{array}{l}\text { No of subject } \\
\text { with TC:Alb }\end{array}$} \\
\hline & $<225$ & $>225$ & $<50$ & $>50$ \\
\hline $\begin{array}{l}<5(n=79) \\
>5(n=43)\end{array}$ & $\begin{array}{r}43 \\
7\end{array}$ & $\begin{array}{l}36 \\
36\end{array}$ & $\begin{array}{r}69 \\
4\end{array}$ & $\begin{array}{l}10 \\
39\end{array}$ \\
\hline
\end{tabular}

Table 2 Comparison of sensitivity and specificity of cholesterol versus cholesterol: albumin ratio using $T C: H D L-C$ as a standard

\begin{tabular}{lcl}
\hline Parameter & \multicolumn{2}{c}{ Based on the TC:HDL-C ratio } \\
\cline { 2 - 3 } & $\begin{array}{l}\text { Subjects correctly } \\
\text { classified }\end{array}$ & $\begin{array}{l}\text { Subjects incorrectly } \\
\text { classified }\end{array}$ \\
\hline $\begin{array}{l}\text { Total cholesterol } \\
\begin{array}{l}\text { Cholesterol: } \\
\text { albumin ratio }\end{array}\end{array}$ & $79 / 122(64.4 \%)$ & $43 / 122(35.6 \%)$ \\
\hline
\end{tabular}

Table 1 shows the TC and TC:Alb ratio compared in their abilities to differentiate subjects with normal $(<5)$ and increased $(>5)$ TC:HDL-C ratios. In the subjects with TC:HDL-C less than $5,36 / 79(43 \%)$ individuals had a TC of $>225 \mathrm{mg} / \mathrm{dl}$. This figure was reduced to $10 / 79(12 \%)$ when the TC:Alb ratio was used. In subjects with a TC:HDL-C ratio of $>5$, the TC:Alb ratio is less useful since in most cases, the concentrations of serum TC are high. Table 2 shows the comparison of the overall sensitivity and specificity of TC versus TC:Alb. The TC:Alb ratio has better sensitivity and specificity than TC $(p<0.001)$. If one regards a TC:HDL-C of $>5$ as "disease", then using TC $>225 \mathrm{mg} / \mathrm{dl}$ as a positive screening test result, this test has a sensitivity of $36 / 43(0.837)$ and specificity of $43 / 79(0.544)$. Using the alternate screen of TC:Alb with designation of TC: Alb $>50$ as a positive screening test, this test has a sensitivity of $39 / 43(0.907)$ and specificity of $69 / 79(0 \cdot 873)$. Hence the TC:Alb ratio at the particular cut-off chosen has both better sensitivity and specificity.

\section{Discussion}

We are not aware of the correlation between serum albumin and HDL-C being previously documented, and serum albumin concentrations have never been shown to have a predictive value for coronary heart disease. Based on this relation between HDL-C and albumin, a total cholesterol:albumin ratio was used to discriminate between subjects with normal and increased TC:HDL-C. The TC:Alb ratio performed better than TC by itself. However, it is important to note that the high correlation between TC:Alb and 
TC:HDL-C is partly due to the total cholesterol forming the numerator of both ratios. The TC:HDL-C ratio is most helpful in distinguishing patients with a modest hypercholesterolaemia due to increased HDL from patients whose hypercholesterolaemia is due to increased light density lipoprotein. HDL-C measurement is usually necessary to make this distinction. Because increased costs are incurred in HDL-C measurement, the use of the TC:Alb ratio would help in considerably reducing the number of HDL-C determinations necessary. Both TC and albumin are routinely available on multichannel analysers. We believe that the ratio is useful only in those subjects with modest hypercholesterolaemia since individuals with low or very high cholesterol concentrations are unlikely to have their risk of developing CHD altered either by HDL-C measurement or the calculation of the ratio. $^{7}$

The explanation for the correlation between HDL-C and serum albumin may be related to the fact that lysolecithin, one of the products of the lecithin cholesterol acyltransferase (LCAT) reaction, is removed by binding to serum albumin. ${ }^{8}$ Since LCAT is implicated in the production of HDL cholesterol esters, increased amounts of albumin may be necessary to remove an increased amount of lysolecithin formed as a result of increased HDL and HDL-cholesterol ester formation.

Before our suggested ratio is applied, it is clear that a large epidemiological study would be neces- $\stackrel{0}{-\frac{0}{*}}$ sary to determine whether the TC:Alb ratio has a better predictive value in terms of morbidity and mortality than total cholesterol alone.

\section{References}

' Castelli WP, Doyle JT, Gordon T, et al. HDL cholesterol and $\mathbb{\Phi}$ other lipids in coronary heart disease. Circulation w 1977;55:767-72.

${ }^{2}$ Kannel WB, Castelli WP, Gordon T. Cholesterol in the predic $\vec{\circ}$ tion of atherosclerotic disease. Ann Intern Med 1979;90:8591.

${ }^{3}$ Gordon T, Castelli WP, Hjortland MC, Kannel WB, Dawbes TR. High density lipoprotein as a protective factor agains? coronary heart disease. Am J Med 1977;62:707-14.

${ }^{4}$ Castelli WP. Cardiovascular statistics in the United States. In Long C, ed. Prevention and rehabilitation in ischaemic heares disease. Baltimore: Williams and Wilkins Co. 1980:1-17.

${ }^{5}$ Glueck CJ, Gartside P, Fallat RW, Sielski J, Steiner PM. Longev- $\vec{\sigma}$ ity syndromes: familial hypobeta and familial hyperalpha $\mathrm{O}$ lipoproteinemia. J Lab Clin Med 1976;88:941-57.

${ }^{6}$ Lipid Research Clinics Program Manual Operation, Vol $\mathbf{1}_{r}$ Washington DC, DHEW Publication No. (NIH) 75-628 1974.

' Connor WE, Connor SL. Dietary treatment of hyperlipidemia In: Rifkind BM, ed. Hyperlipidemia, diagnosis and therapy. New York: Grune and Stratton, 1977: chapter 13.

${ }^{8}$ Tall AR, Small DM. Plasma high-density lipoproteins $N$ Engl $j$ Med 1978;299:1232-6.

Requests for reprints to: Dr Amin A Nanji, Division o Clinical Chemistry, Vancouver General Hospital, 8550 West 12th Avenue, Vancouver, Canada V5Z 1M9. 